\title{
QUISTE HIDATÍDICO INTRACARDÍACO EN UNA NIÑA: REPORTE DE CASO
}

\author{
Anderson V. Huerta-Obando ${ }^{1,2, a}$, Erick Y. Olivera-Baca ${ }^{2, b}$, Jaime Silva-Díaz ${ }^{2, b}$, \\ Abel Salazar-Díaz ${ }^{2, b}$
}

\begin{abstract}
RESUMEN
La equinococosis es una infección parasitaria provocada por Echinococcus granulosus, que, en su estado quístico, forma al denominado quiste hidatídico. Presenta morbilidad importante, con posibles secuelas relacionadas con la ubicación, y altos costos debido al tratamiento quirúrgico y farmacológico prolongado. El hígado y el pulmón son las ubicaciones anatómicas más usuales, mucho más raras son el riñón, bazo, cerebro y corazón, este último representa el $0,5 \%$ a $2 \%$ del total de casos. El Perú es un país endémico de esta antropozoonosis y principalmente registra casos procedentes de la sierra central $(95 \%)$. Se presenta el caso de una niña de diez años, con diagnóstico de esta entidad, clasificación ecográfica CE 1, grupo clínico 1 (confirmado por anatomía patológica) con posterior tratamiento quirúrgico y farmacológico específico (albendazol). La paciente se recuperó satisfactoriamente de la cirugía practicada, y fue dada de alta a los 16 días, sin complicaciones.
\end{abstract}

Palabras clave: Equinococosis; Cirugía torácica; Corazón; Tabique interventricular; Niño (fuente: DeCS BIREME).

\section{CARDIAC HYDATID CYST IN A CHILD: A CASE REPORT}

\begin{abstract}
Echinococcosis is a parasitic infection caused by Echinococcus granulosus, which, in its cystic state, forms the socalled hydatid cyst. It presents important morbidity, with possible sequelae related to the location, and high costs due to surgical and prolonged pharmacological treatment. The liver and the lung are the most common anatomical locations, and much rarer are the kidney, spleen, brain, and heart, where the latter represents 0.5 to $2 \%$ of total cases. Peru is an endemic country of this anthropozoonosis and mainly records cases in the central highlands (95\%). This paper presents the case of a 10-year-old girl, diagnosed with this disease, CE1 ultrasound classification, clinical group 1 (confirmed by pathological anatomy) with specific surgical and pharmacological treatment (albendazole) afterward. The patient recovered satisfactorily from the surgery and was discharged at 16 days, without complications.
\end{abstract}

Keywords: Echinoccocosis; Thoracic surgery; Heart; Ventricular septum; Child (source: MeSH NLM).

\section{INTRODUCCIÓN}

La hidatidosis o equinococosis quística (unilocular), término que deriva del griego «hydatidos» que significa «vejiga con agua», es una antropozoonosis provocada por el estado larval del Echinococcus granulosus, y el quiste hidatídico, por el estado quístico del mismo ${ }^{(1,2,5-7)}$. Los quistes hidatídicos se localizan principalmente en hígado ( $65 \%$ de los casos) y en pulmones $(25 \%)^{(1-3)}$. Otras localizaciones representan el $10 \%{ }^{(5)}$. El compromiso del corazón es infrecuente ${ }^{(2,5)}$. La relación en cuanto al sexo es indistinta ${ }^{(2,3)}$. Está relacionada a actividades ganaderas (crianza de ovinos, porcinos y caprinos), deficiente infraestructura, educación sanitaria y nivel socieconómico bajo (carencia de agua potable) ${ }^{(3)}$.
Presenta una morbilidad importante, que puede cursar con síndromes clínicos graves con desenlace fatal si no se tratan; incluso con tratamiento, a menudo disminuye la calidad de vida. La mortalidad posoperatoria media es del $2,2 \%$, y en un $6,5 \%$ de los casos recidivan tras la cirugía, lo que producen periodos prolongados de recuperación. Las secuelas dependerán de la ubicación del quiste ${ }^{(12)}$.

La importancia del diagnóstico y manejo precoz de la equinococosis, junto con la prevención, evitan elevadas tasas de mortalidad, discapacidad, y mayores gastos presupuestales estatales y familiares, ya que el tratamiento resulta caro y complicado debido a requiere cirugía y/o tratamiento farmacológico prolongado. En el 2015, la equinococosis a nivel mundial causó 19300 muertes y pérdida de 871000 años de vida ajustados en función de la discapacidad ${ }^{(12)}$.

\footnotetext{
Facultad de Medicina, Universidad Nacional Mayor de San Marcos. Lima, Perú

Servicio de Clínica Pediátrica, Hospital Nacional Guillermo Almenara Irigoyen. Lima, Perú

Médico residente de Pediatría; ${ }^{\mathrm{b}}$ médico pediatra

Recibido: 19/11/2017 Aprobado: 09/05/2018 En línea: 03/07/2018
}

Citar como: Huerta-Obando AV, Olivera-Baca EY, Silva-Díaz J, Salazar-Díaz A. Quiste hidatídico intracardíaco en una niña: reporte de caso. Rev Peru Med Exp Salud Publica. 2018;35(2):338-43. doi: 10.17843/rpmesp.2018.352.3258. 
E. granulosus, es un metacéstodo ciclofilideo del género Echinococcus de la familia Taeniidae que infecta el intestino del perro (tenia adulta), su huésped definitivo asintomático. En el hombre, su huésped intermediario incidental, un $10 \%$ de sus huevos alcanzan la circulación, llegando a órganos específicos como los pulmones y otras ubicaciones sistémicas como el corazón, páncreas, bazo y cerebro $(2,3,14,10)$.

La llegada de este parásito a las cavidades cardiacas ocurre cuando los embriones hexacantos alcanzan la circulación sistémica y al miocardio a través de la circulación coronaria, arteria pulmonar o foramen oval permeable ${ }^{(14)}$. Una vez que el parásito alcanza al miocardio el quiste se forma entre uno a cinco años ${ }^{(2,5)}$. El ventrículo izquierdo, al recibir más flujo sanguíneo, se compromete con más frecuencia (55\% a $60 \%)$, seguido del ventrículo derecho (15\% a $25 \%)$, la aurícula izquierda ( $8 \%$ ), el pericardio ( $8 \%$ a $11 \%$ ), el septum interventricular (5\% a $9 \%$ ) y arterias pulmonares $(7 \%)$ por su mayor vascularización ${ }^{(2-5,10)}$. Los quistes hidatídicos del ventrículo izquierdo son usualmente localizados en el subepicardio, mientras que los del septum interventricular son intramiocárdicos ${ }^{(3,5,14)}$.

Esta entidad tiene distribución mundial, ha sido registrada en todos los continentes, excepto en la Antártida. Sudamérica notifica anualmente 2000 casos de hidatidosis, donde es considerada endémica ${ }^{(2,3,5,7)}$. Según estadísticas al 2017 de la Organización Mundial de la Salud (OMS), la prevalencia alcanza de $5 \%$ a $10 \%$ en algunas zonas de Argentina, Perú, África Oriental, Asia Central y China. En zonas endémicas las tasas de incidencia en humanos pueden exceder 50 por 100000 años-persona ${ }^{(12)}$.

En Perú, la sierra central posee el $95 \%$ de los casos reportados ${ }^{(5)}$, en el 2012, la prevalencia de hidatidosis humana osciló entre 7 a 11 por 100000 habitantes. Sin embargo, en Huancavelica, Cerro de Pasco, Ayacucho e Ica, oscila entre 14 - 34 por 100000 habitantes $^{(3)}$.

Se reporta el caso de una niña con quiste hidatídico intracardiaco, diagnosticada y tratada en el Hospital Nacional Guillermo Almenara Irigoyen en Lima, Perú. Esta publicación resalta la ubicación poco frecuente del quiste, el diagnóstico y manejo específico.

\section{REPORTE DE CASO}

Niña de 10 años, natural y procedente de Lima (distrito de Independencia), raza mestiza, estudiante del sexto grado de primaria, nunca residió en otro lugar. Ingresó a la emergencia pediátrica del Hospital Nacional Guillermo Almenara Irigoyen en mayo de 2016, con un tiempo
Tabla 1. Características laboratoriales seriadas de los hemogramas completos y proteína $\mathrm{C}$ reactiva.

\begin{tabular}{lccc}
\hline Examen Auxiliar & Ingreso & Día 8 & Día 10 \\
\hline Proteína C Reactiva & 10 & - & - \\
\hline Leucocitos (cel/ml) & 8610 & 14670 & 11890 \\
\hline Abastonados (\%) & 0 & 12 & 0 \\
\hline Segmentados (\%) & 62,9 & 76 & 75 \\
\hline Eosinófilos (\%) & 3,4 & 0,1 & 0,2 \\
\hline Linfocitos (\%) & 28,2 & 7,9 & 14,9 \\
\hline Hemoglobina (g/dL) & 12,7 & 11,2 & 10,5 \\
\hline Hematocrito (\%) & 38,2 & 33,1 & 31,9 \\
\hline Plaquetas (cel/uL) & 242000 & 190000 & 156000 \\
\hline
\end{tabular}

de enfermedad de dos meses, inicio insidioso y curso progresivo, caracterizado por disnea a grandes esfuerzos y palpitaciones esporádicas que cedían con el reposo. Una semana antes del ingreso, presentó incremento de palpitaciones que no cedían al reposo, asociado a dolor torácico de tipo opresivo, además de cefalea global que disminuyó parcialmente con paracetamol. No presentó fiebre, ni cianosis y negó viajes realizados en el último año.

Como antecedentes personales, la paciente fue hospitalizada a los tres años por convulsión febril por foco faríngeo. A los ocho años presento convulsión por traumatismo craneo-encefálico (caída de escaleras). Cirugía a los cuatro años por hernia inguinal derecha. Todas sus inmunizaciones están completas. Reside en vivienda de adobe y piso de tierra, el abastecimiento de agua es por medio de cisterna y la eliminación de excretas por silo. Tiene animales domésticos: un perro, tres gatos, y 30 cuyes (contacto desde los cuatro años), ademas de roedores e insectos en casa. Sus familiares aparentemente sanos, sin historia previa de patología cardiaca, ni de enfermedades genéticas.

Al examen físico, la paciente presentó una frecuencia cardiaca de 41 latidos/minuto, el resto de funciones vitales, incluida la saturación de oxígeno, fueron normales. Regular estado general, hidratación y nutrición. A nivel cardiovascular: precordio normodinámico, no choque de punta, ruidos cardiacos arrítmicos, bradicárdicos, buena intensidad, primer y segundo ruidos conservados, soplo sistólico II/NI borde esternal izquierdo, pulsos periféricos presentes y simétricos. A nivel cervical no se encontró ingurgitación yugular. Resto del examen físico dentro de rangos normales.

Los exámenes auxiliares laboratoriales al ingreso se describen en la tabla 1. Otros exámenes realizados se detallan a continuación: 
Electrocardiograma (Emergencia): Ritmo sinusal auricular. Frecuencia cardiaca: 45 latidos/minuto. QRS: $120 \mathrm{~ms}$ (prolongado), eje QRS: $+120^{\circ}$, disociación aurículoventricular, bloqueo completo aurículoventricular de III grado.

Radiografía de tórax (Emergencia): crecimiento ventrículo izquierdo, índice cardiotorácico: 0,51, flujo pulmonar normal.

Ecocardiografía (día dos de hospitalización): Imagen quística 6,6 x 4,7 x 4,6 cm con crecimiento hacia ventrículo izquierdo (VI) dependiente del septum interventricular que lo desplaza hacia ventrículo derecho con dilatación leve de VI con función sistólica conservada, foramen oval permeable $2 \mathrm{~mm}$.

Tomografía tóracoabdominal (día dos de hospitalización): tumoración quística única en corazón de $72 \times 50 \mathrm{~mm}$, tumoración quística de $35 \times 27 \mathrm{~mm}$ y otra de $35 \times 31 \mathrm{~mm}$ en lóbulo derecho hepático, no evidencia de quiste en pulmón (Figura 1).

En el segundo día hospitalización, luego de observar la lesión quística intracardiaca e intrahepática, se decide iniciar tratamiento con albendazol $10 \mathrm{mg} / \mathrm{kg} / \mathrm{día}$. Posteriormente, en el cuarto día de hospitalización, los exámenes laboratoriales específicos mostraron IgG E. granulosus positivo, IgG Cisticerco negativo, e Inmunoblot hidatidosis negativo.

Enel octavo día de hospitalización, se realiza la cirugía cardíaca consistente en esternotomía media, pericardiectomía, canulación aorto-bicaval, ingreso a circulación extracorpórea (CEC), atriotomía. Al identificar los hallazgos se procedió a inyección de solución hipertónica al $20 \%$, y posteriormente exéresis del quiste intracardíaco más plicatura de cavidad. Luego de salida de CEC, se colocó un marcapaso epicárdico definitivo. El hallazgo operatorio fue una tumoración quística de $5 \mathrm{~cm} \times 5 \mathrm{~cm}$ en septo interventricular, de contenido claro, cobertura y membrana blanca lisa nacarada, no se evidencia compromiso valvular. El resultado de anatomía patológica confirmó el diagnóstico de quiste hidatídico intracardiaco (Figura 2).

Durante el postoperatorio, la paciente ingresa a la Unidad de Cuidados Intensivos Cardiovascular por ocho días, debido a que presentó derrame pleural y atelectasia, los cuales remitieron al alta. Las evaluaciones ambulatorias mostraron evolución favorable, manteniendo frecuencias cardiacas estables de 90 latidos/minuto, sin inestabilidad hemodinámica ni bloqueo auriculoventricular. Como secuela se halló quiste intracardiaco residual, insuficiencia mitral moderada y disfunción cardiaca del ventrículo izquierdo leve. Fue manejado con hidroclorotiazida y captopril, fisioterapia cardiaca y respiratoria, además de cuatro ciclos de albendazol (10mg/kg/día) vía oral. No se observó ninguna reacción adversa medicamentosa.

\section{DISCUSIÓN}

La localización del quiste hidatídico a nivel cardiaco es muy rara; se reporta $0,5 \%$ a $2 \%$ de los casos $(2,3,9,10)$. Otros reportan frecuencias del $0,041 \%$ a $0,37 \%(4,11)$. Más del $50 \%$ de estos casos intracardiacos presentan compromiso multiorgánico ${ }^{(5)}$.

En el Perú, se han reportado tres casos de hidatidosis intracardiaca. En el 2005, Montero et al., reportan un paciente adulto varón de 21 años con un quiste intracardiaco único a nivel del septum interventricular (3). En el 2010, Ramirez et al., reportan una paciente mujer obesa de 45 años con un quiste hidatídico a nivel del vértice cardiaco izquierdo de $73 \times 57 \mathrm{~mm}$, y otro a nivel hepático ${ }^{(4)}$. En el 2012, Castillo et al., reportan un paciente varón de once años con un quiste intracardiaco de 35,2 x $31 \mathrm{~mm}$ ubicado en la pared postero lateral del ventrículo izquierdo, y otro quiste pulmonar basal izquierdo (2).

La presentación clínica del quiste intracardiaco es inespecífica, varía de acuerdo al tamaño, lugar y efecto de masa del quiste, pudiendo ser asintomáticos (10\%) y otros al crecer, desplazan y adelgazan la pared ventricular entre el epicardio hacia el endocardio, pueden presentar angina secundaria a compresión coronaria, dolor precordial o retroesternal, disnea y palpitaciones ${ }^{(3,5,6,9,11)}$

Se reportan eventos isquémicos, lesión valvular, trastornos de la conducción (arritmias), hipertensión pulmonar, desprendimiento de los componentes estructurales de la superficie quística formando microémbolos (sobre todo cerebral), ruptura quística extracavitaria (ventrículo izquierdo) e intracavitaria (ventrículo derecho), efecto de masa provocando insuficiencia cardiaca, pericarditis aguda o taponamiento cardiaco, reacciones anafilácticas y shock ${ }^{(3,4,5,9,10)}$. La estimación del aumento medio del diámetro del quiste varía aproximadamente de 1 a 1,5 cm/año, por ello son muy raros en niños ${ }^{(3,5)}$. El tiempo estimado para la aparición del quiste luego de la infección es de diez meses ${ }^{(5)}$.

El diagnóstico de la hidatidosis intracardiaca se basa en la historia clínica epidemiológica, exámenes serológicos, y la visualización del quiste por métodos de apoyo diagnóstico (dada la baja especificidad de las pruebas de laboratorio para equinococosis cardiaca), donde el ecocardiograma permanece como el método de imagen más fiable, más rentable y de mayor alcance; sin embargo, también la resonancia magnética y tomografía son los principales métodos de imagen, ya que proporcionan resultados más precisos, delimitantes para la extirpación quirúrgica exacta de los quistes ${ }^{(3,5,8,10)}$

La OMS ha elaborado una clasificación ecográfica de quistes hidatídicos hepáticos, que ha sido extrapolada para 

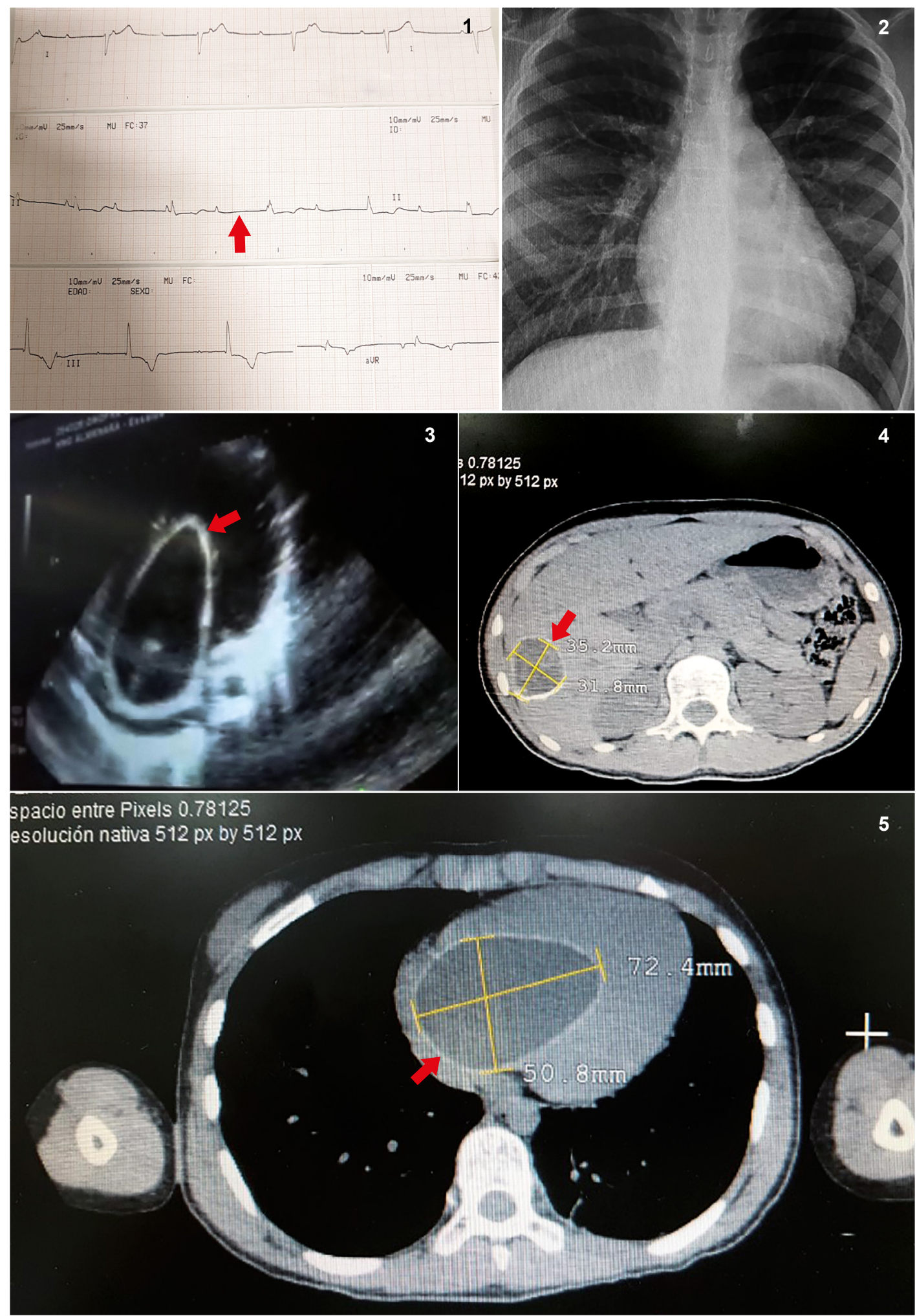

Figura 1. Exámenes auxiliares electrocardiográfico y de imágenes de apoyo diagnóstico (radiografía, ecocardiografía, tomografía).

(1) Electrocardiografía de ingreso muestra bloqueo A-V III grado. (2) Radiografía de tórax al ingreso sin evidencia de imagen quística. (3) Ecocardiograma revela imagen quística intracardiaca dependiente del septum interventricular (4) Tomografía abdominal muestra imagen quística de $31 \times 35 \mathrm{~mm}$ en lóbulo hepático derecho. (5) Tomografía torácica muestra imagen quística intracardiaca de $72 \times 50 \mathrm{~mm}$. 


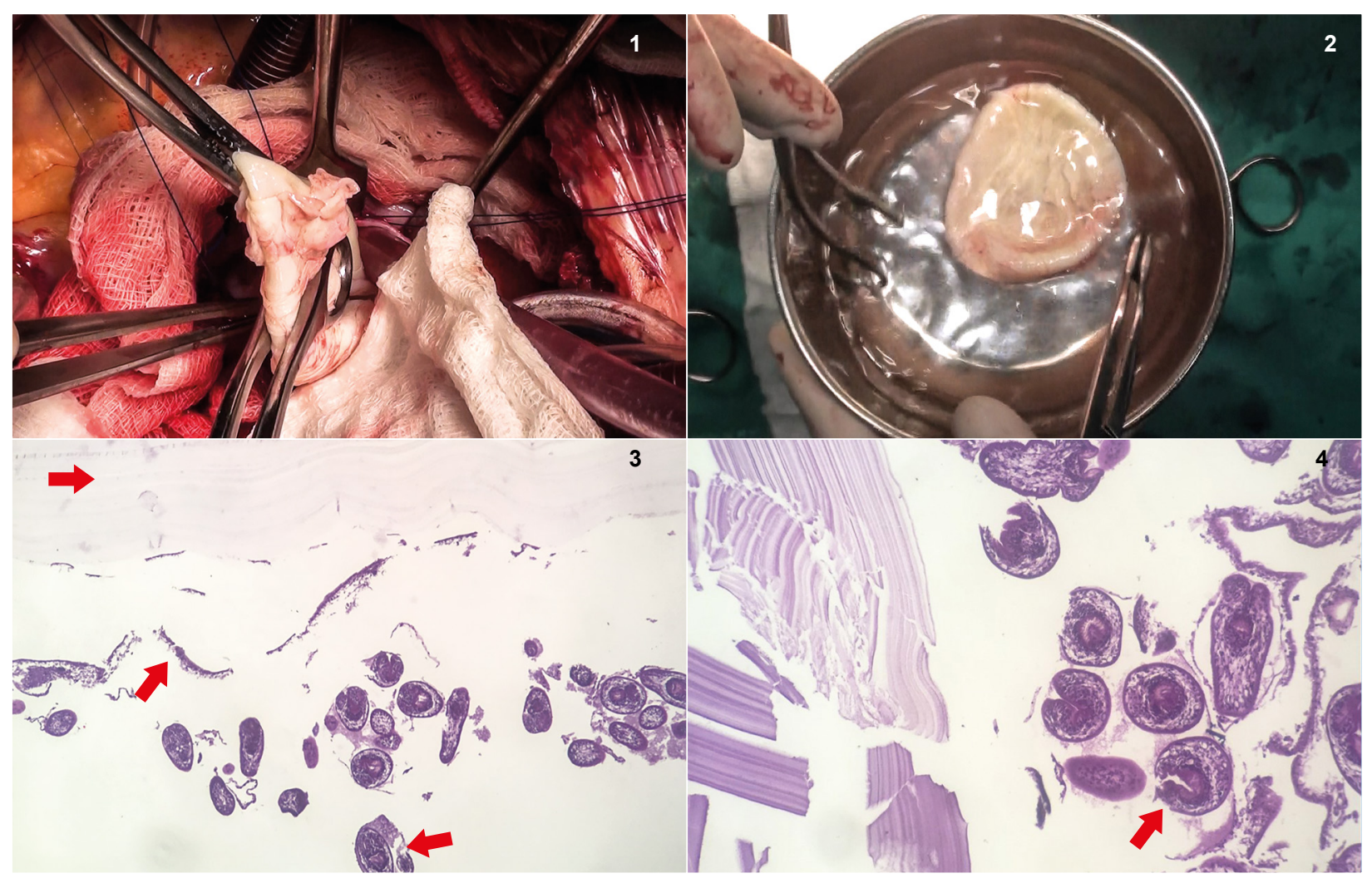

Figura 2. Imágenes de cirugía cardiaca (exéresis de quiste) y láminas anatomopatológicas de pieza quirúrgica extraída

(1) Cirugía cardiaca abierta que muestra exéresis de quiste. (2) Imagen de pieza anatomopatológica de forma circular y superficie blanco nacarada. (3) Lámina con corte de pieza extraída muestra capa adventicia, capa germinativa y vesículas prolígeras (aumento 2X) (4) Lámina con corte de pieza extraída muestra a mayor aumento las vesículas hijas con escólex en su interior (aumento 3X).

quistes intracardiacos, dividiéndolos en seis categorías y tres grupos clínicos. En el presente reporte se clasifica como quiste hidatídico CE1, grupo clínico $1^{(10)}$. La serología es más sensible y específica para enfermedad hidatídica hepática que para enfermedad hidatídica cardiaca. La prueba de ELISA es la más sensible (94\%) y específica (99 \%) para la mayoría de localizaciones del quiste hidatídico. En la Hidatidosis avanzada existe un aumento marcado del anticuerpo lgG ${ }^{(3,5)}$. La ausencia de serología positiva para el Equinococcus no descarta la enfermedad (50\% de falsos negativos para quistes cardiacos) ${ }^{(7)}$. En nuestro reporte se presentó lgG E. granulosus positivo, pero Inmunoblot negativo. El diagnóstico confirmatorio es la anatomía patológica. El diagnóstico diferencial incluye tumores cardiacos (mixomas), aneurismas del seno de Valsalva, aneurisma de la pared del ventrículo izquierdo y los quistes congénitos del pericardio ${ }^{(10,15)}$.

El tratamiento del quiste hidatídico se debe individualizar y valorar la cirugía, acompañada o no de manejo farmacológico, albendazol, sobre todo en casos de afectación multivisceral o producida por siembra tras rotura de quiste ${ }^{(3,7)}$. El albendazol (10 a $15 \mathrm{mg} / \mathrm{Kg} /$ día) es usado como tratamiento único en quistes pequeños (diámetro menor o igual a cinco $\mathrm{cm}$ ) o calcificados, y como tratamiento complementario a la cirugía. Son necesarios varios ciclos de tratamiento (dos a cinco ciclos) para garantizar la erradicación total del parásito, pudiendo administrarse entre una a cuatro semanas antes de la cirugía. En nuestro paciente se administraron cuatro ciclos posteriores a la cirugía ${ }^{(3,6,7,14)}$. El quiste hidatídico cardíaco debe ser removido quirúrgicamente, incluso en pacientes asintomáticos ${ }^{(5,8)}$.

Nohayconsenso con respectoal manejo de la cavidad residual después de retirar la membrana hidatídica (autocierre por segunda intención, capitonaje). Las secuelas posquirúrgicas incluyen desgarro miocárdico, bloqueo auriculoventricular, arritmias ventriculares y muerte súbita ${ }^{(2,10)}$.

En conclusión, la hidatidosis intracardiaca se puede presentar con variedad clínica; la sospecha diagnóstica ante cualquier tumor quístico intracardiaco debe estar siempre presente en países endémicos, y se debe realizar precozmente el tratamiento específico (quirúrgico y farmacológico), así como actividades de prevención debido a los altos costos, alto riesgo de complicaciones, y posibles secuelas discapacitantes, asegurando el seguimiento de la cavidad residual y secuelas posquirúrgicas. 
Aspectos éticos: Para la publicación del presente reporte de caso se contó con el consentimiento de los padres del paciente.

Agradecimientos: A todos los médicos residentes y asistentes del servicio de Clínica Pediátrica y Cirugía Cardiovascular del Hospital Nacional Guillermo Almenara Irigoyen, quienes con su dedicación, cuidados y experiencia garantizaron el manejo óptimo de nuestra paciente.

Contribución de autoría: AVHO ha participado en la concepción y diseño del artículo, la recolección, análisis e interpretación de datos, redacción del artículo, la revisión crítica del artículo y aprobación de la versión final. EYOB ha participado en la recolección, análisis e interpretación de datos, redacción del artículo, la revisión crítica del artículo y aprobación de la versión final. JSD y ASD han participado en el análisis e interpretación de datos, redacción del artículo, la revisión crítica del artículo y aprobación de la versión final.

Fuente de financiamiento: Autofinanciada.

Conflicto de interés: Los autores declaran no tener conflictos de interés.

\section{REFERENCIAS BIBLIOGRÁFICAS}

1. Salazar P, Cabrera M. Hidatidosis. En: Becerril M. Parasitología Médica. 4ta edición. México: McGraw-Hill/Interamericana Editores; 2014. p. 187-95.

2. Castillo De la Cadena L, Cuba Y, Mendoza C, Mispireta M, Parra J, Pacheco P. Quiste cardiaco hidatídico en un niño: Presentación de un caso. Rev Med Hered. 2012;(23):36-40.

3. Montero F, Contreras O, Barriga E, Curi Y, Cosme P, Delgado C, et al. A propósito de un caso de quiste hidatídico cardiaco. Rev Per Cardiol. 2005;31(3):180-5.

4. Ramírez J, Hernández J, Orlovac A, Málaga G, Cáceres J. Equinococcosis cardiaca y obesidad mórbida. Consideraciones quirúrgicas. Acta Med Per. 2010;(27):123-6.

5. Zárate L, Contreras E. Equinococosis cardiaca. Rev Mex Cardiol. 2010;21(2):57-69.

6. Noemí I, Viovy A, Zamorano R, Blanco A, Revello D, Vojkovic M, et al. Hidatidosis en la infancia: Albendazol en su tratamiento médico y quirúrgico. Rev Chil Infect. 2003;20(4):229-34.

7. García P, Chabes A, Fernández $M$, Guerra MI, Lévano I, Quiroz S, et al. Características clínicas y epidemiológicas de hidatidosis en el Hospital Nacional Dos de Mayo. Rev Med Hum Univ Ricardo Palma. 2006;6(2):26-30.

8. Ozkan M, Astarcioglu M, Tuncer A, Gursoy O, Aykan A. Quiste hidatídico cardiaco: un enfoque multimodal. Rev Esp Cardiol. 2012;65(2):187-95.

9. Slobodan I, Vojislav P, Milan D, Kalangos A. Ruptured hydatid cyst of the interventricular septum with acute embolic pulmonary artery complications. Pediatric Cardiology. 2008;29:855-7.

10. Kahlfuß S, Flieger R, Roepke T, Yilmaz K. Diagnosis and treatment of cardiac echinococcosis. Heart 2016;102:1348-53.

11. Olgun H, Karacan M, Ceviz N, Kocak H. Cardiac Hyatid Cyst. Eurasian J Med. 2009;41(1):73.

12. Equinococosis: Nota descriptiva [Internet]. Organización Mundial de la
Salud. Ginebra: OMS; 2017 [citado 2018 abril 19]. Disponible en http:// www.who.int/mediacentre/factsheets/ fs $377 /$ es/

13. Koneti K, Jagannath B, Sreeram N. Cardiac hydatd cyst. Cardiol Young. 2013;23(4):606-7.

14. Sánchez J, Muratore I, Esteban A, Costantini S, Moyano C. Hidatidosis quística del septum interventricular. Rev Fed Arg Cardiol. 2014; 43(2):103-106.

15. Miranda R, Merchak A, Ferrier P, Villarroel A, Edding O. Quiste hidatídico cardiopericárdico: Presentación de dos casos clínicos. Rev Chil Radiol. 2002;8:123-26.

Correspondencia: Anderson Valentin Huerta Obando

Dirección: AAHH 31 de diciembre Mz. L, Lt.7. Independencia. Lima, Perú

Teléfono: (511) 954704753

Correo electronico: huertaandy@hotmail.com 